\title{
Parameter Optimization of Shot Peening Process of PMG AL2024 Alloy Cover
}

\author{
Mr.Atul Pathak ${ }^{1}$, Prof Dr Kadam Munjadas ${ }^{2}$ \\ Department of Mechanical Engineering, Jawaharlal Nehru Engineering College, Aurangabad, India ${ }^{l}$ \\ Department of Mechanical Engineering, Jawaharlal Nehru Engineering College, Aurangabad, India ${ }^{2}$
}

\begin{abstract}
Shot peening leads to local plastic deformations in the near-surface regions, which result in the development of compressive residual stress and the improvement of surface hardness in the aerospace structural components. These properties can be enhanced by careful selection of the peening parameters. PMG Cover of AL2024 Aluminum Alloy is widely used in the generator manufacturing cover due to its high specific static strength. In this study a Taguchi Grey Relational Analysis is presented to optimize the surface properties of residual stress, micro hardness. The effects of four peening parameters (Shot Diameter, Shot Velocity, Impact Angle, Nozzle Distance) on micro hardness and residual stress are investigated Design of Experiment work is carried out by MINITAB 14 software tools of Taguchi Grey relational method, for getting excellent shot peening process parameter combination by MAT LAB R2009 software tools of advanced Optimization method as Genetic Algorithm, Simulated Annealing. Compare of the above reading for the investigation.
\end{abstract}

Keywords: Compressive Residual Stresses, Design of Experiments, Genetic Algorithm, Grey Relation Analysis, Simulated Annealing.

\section{Introduction to DOE}

Experiments are performed by investigators in virtually all fields of inquiry, usually to discover something about a particular process or system. Literally, an experiment is a test. More formally, we can define an experiment as a test or series of tests in which purposeful changes are made to the input variables of the process or system so that we can observe and identify the reasons for changes that may be observed in output response. In engineering, experimentation plays an important role in new product design, manufacturing process development and process improvement. The objective in many cases may be to develop a robust process that is a process affected minimally by external sources of variability. Any scientific investigation involves formulation of certain assertions (or hypotheses) whose validity is examined through the data generated from an experiment conducted for the purpose. Thus experimentation becomes an indispensable part of every scientific end designing an experiment is an integrated component of every research program. Three basic techniques fundamental to designing an experiment are replication, local control (blocking), and randomization. Whereas the first two help to increase precision in the experiment, the last one is used to decrease bias. These techniques are discussed briefly below.

Replication is the repetition of the treatments under investigation to different experimental units. Replication is essential for obtaining a valid estimate of the experimental error and to some extent increasing the precision of estimating the pair wise differences among the treatment effects. It is different from repeated measurements. Suppose that the four animals are each assigned to a feed and a measurement is taken on each animal. The result is four independent observations on the feed. This is replication. On the other hand, if one animal is assigned to a feed and then measurements are taken four times on that animal, the measurements are not independent. We call them repeated measurements. The variation recorded in repeated measurements taken at the same time reflects the variation in the measurement process, while variation recorded in repeated measurements taken over a time interval reflects the variation in the single animal's responses to the feed overtime. Neither reflects the variation in independent animal's responses to feed. We need to know about the latter variation in order to generalize any conclusion about the feed so that it is relevant to all similar animals.

For inferences to be broad in scope, it is essential that the experimental conditions should be rather varied and should be representative of those to which the conclusions of the experiment are to be applied. However, an unfortunate consequence of increasing the scope of the experiment is an increase in the variability of response. Local control is a technique that can often be used to help deal with this problem.Blocking is the simplest technique to take care of the variability in response because of the variability in the experimental material. To block an experiment is to divide, or partition, the observations into groups called blocks in such a way that the observations in each block are collected under relatively similar experimental conditions. If blocking is done well, the comparisons of two or more treatments are made more precisely than similar comparisons fro man unblocked design. 
The purpose of randomization is to prevent systematic and personal biases from being introduced into the experiment by the experimenter. A random assignment of subjects or experimental material to treatments prior to the start of the experiment ensures that observations that are favored or adversely affected by unknown sources of variation are observations "selected in the luck of the draw" and not systematically selected.Lack of a random assignment of experimental material or subjects leaves the experimental procedure open to experimenter bias. For example, a horticulture is may assign his other favorite variety of experimental crop to the parts of the field that look the most fertile, or a medical practitioner may assign his or her preferred drug to the patients most likely to respond well. The preferred variety or drug may appear to give better results no matter how good or bad it actually is Lack of random assignment can also leave the procedure open to systematic bias. Consider, for example an experiment conducted to study the effect of drugs in controlling the blood pressure. There are three drugs available in the market that can be useful for controlling the diastolic blood pressure. There are 12 patients available for experimentation. Each drug is given to four patients. If the allotment of drugs to the patients is not random, then it is quite likely that the experimenter takes four observations on drug 1 from the four patients on whom the onset of the disease is recent the four observations on drug 2 are taken on four patients on whom the disease is 5-6 years old and the four observations on drug 3 are taken on four patients on whom the disease is chronic in nature.

This arrangement of treatments on patients is also likely if the assignment of drugs to the patients is made randomly. However, deliberately choosing this arrangement could well be disastrous. Duration of illness could be a source of variation and, therefore, response to drug 1 would be better as compared to drug 2 and drug 3. This could naturally lead to the conclusion that drug 1 gives a better response to control blood pressure as compared to drug 2 and drug 3 There are also analytical reasons to support the use of a random assignment. The process of randomization ensures independence of observations, which is necessary for drawing valid inferences by applying suitable statistical techniques. It helps in making objective comparison among the treatment effects.

\subsubsection{Steps in DOE:}

\section{a) Planning phase:}

A designer learned something from each experiment that will positive or negative. Positive information is an indication of which factor and which levels leads to improved process performance. Negative information is an indication of which factors don't lead improvement, but no indication of which factors do.

b) Conducting phase:

In this phase, test results are actually collected. If experiments are well planned and conducted then analysis is easier and give positive information about factors and levels.

\section{c) Analysis phase:}

In analysis phase positive or negative information regarding factors and levels are generated. This phase is most statistical in nature of the three phases of the DOE.

\subsection{Design of Experiments (DOE) By Taguchi Method}

Design of Experiments (DOE) is a powerful statistical technique introduced by R. A. Fisher in England in the 1920's to study the effect of multiple variables simultaneously. In his early applications, Fisher wanted to find out how much rain, water, fertilizer, sunshine, etc. are needed to produce the best crop. Since that time, much development of the technique has taken place in the academic environment, but did help generate many applications in the production floor. As a researcher in Electronic Control Laboratory in Japan, Dr. Genechi Taguchi carried out significant research with DOE techniques in the late 1940's. He spent considerable effort to make this experimental technique more user-friendly (easy to apply) and applied it to improve the quality of manufactured products. Dr. Taguchi's standardized version of DOE, popularly known as the Taguchi method or Taguchi approach, was introduced in the USA in the early 1980's. Today it is one of the most effective quality building tools used by engineers in all types of manufacturing activities. The DOE using Taguchi approach can economically satisfy the needs of problem solving and product/process design optimization projects. By learning and applying this technique, engineers, scientists, and researchers can significantly reduce the time required for experimental investigations.

The purpose of process development is to improve the performance characteristics of the process relative to customer needs and expectations. Taguchi addresses quality in two main areas: off line and on line quality control. Both of these areas are very cost sensitive in the decisions that are made with respect to the activities in each. Off line quality control refers to the improvement of quality in the process development stages. On line quality control refers to the monitoring of current manufacturing process to verify the quality levels produced. [P.J. Ross, 1996]To achieve desirable product quality by design, Taguchi views the design of a process as a three phase program. These are as follows.

a) System design:

System design is the phase where new concepts, ideas, methods are generated and that provide new product to customers. 
b) Parameter design:

In this phase, best setting for control parameters are determined that not affect manufacturing cost and the setting that minimize quality loss.

c) Tolerance design:

Tolerance design phase improves quality at minimal cost. Quality is improved by tighten tolerance on process parameters to reduce the performance deviation.

1.2.1 Signal to Noise (S/N) Ratio:

Taguchi developed $\mathrm{S} / \mathrm{N}$ ratio as a proactive equivalent to the reactive loss function. Signal factors are the set by the designer or operator to obtain the intended value of the response variable. Noise factors are not controlled or are very expensive or difficult to control

\section{Nominal the Best S/N Ratio:}

It is used wherever there is a nominal or target value and a variation about that value such as dimension, voltage, weight and so forth. The target value is finite but not zero. For robust design, $\mathrm{S} / \mathrm{N}$ ratio should be maximized. The nominal is best value is a maximum when average is large and variance is small. When average is off target on high side, $\mathrm{S} / \mathrm{N}$ ratio value can give more favorable information.

\section{Smaller the better:}

This ratio is used when target value is zero such as response time of computer, emission from automobile and corrosion etc.

3. Larger the better:

It is used where the largest target value is desired such as weld strength, mileage or yield of process.

\subsubsection{Steps for Taguchi Design of Experiment:}

1. State the problem(s) or area(s) of concern:

In this step the problem is stated with its objective as well as purpose. Also during this all the necessary information is stated regarding the problem like how the problem is observed, when problem occurs, how severe the problem is etc.In our experiment the problem is stated in chapter number 1 . We have to improve the fatigue life of the material with help of shot peening

2. State the objective(s) of the experiment:

We have state the objective of the experiment. The objective should be specific for better experiment planning. We have to improve the fatigue life of material by identifying the parameters and optimize it.

3. Select the quality characteristics and measurement system:

The quality characteristics are to be stated and how they are to be measured is to be decided. We will measure the residual stresses Finite Element Modeling after that physical experimentation by XRD Diffraction method and fatigue life is to be tested by rotating beam bending machine.

4. Select the factors that may influence the selected quality characteristics:

The factors which influence quality a characteristic is to be identify. Detailing of process is desired. The shot peening will induced residual stress in the specimen. Residual stress will improve the fatigue life. So residual stress is controlling parameter for fatigue life as the characteristic.

As per literature review, Shot size, Shot Velocity, Impact Angle \& Shot Distance are the most influential parameters on residual stresses.

\section{Identifying the Control and Noise factors:}

In this step, all the control factors and noise factors are decided. Noise factors are not controlled or very expensive to control or difficult to control.

We have described the control factors in previous step. Noise factors in shot peening are heat energy produced in the process, wear of shot during the process, unavoidable interference of shots, producing cracks in weak sections.

\section{Select levels of the factors:}

The levels of factors are selected in the experiment. The experiment should be feasible with the given levels. In our particular experiment, 4 levels are selected. Means each factor will have 4 values.

Shot size in $\mathrm{mm}-0.65,0.75,1,1.25$

Shot Velocity in $\mathrm{m} / \mathrm{s}-30,40,45,60$

Shot Impact angle in Degrees- 45, 65, 80, 85

Shot Distance in mm- 90,120,150,190

7. Select the appropriate orthogonal array:

The proper array has to be selected for correct experiment. This will create proper iterations for the experiment. So for 4 Level and 4 Control Factors we selected L16 Orthogonal Array in available option. Fig 1.1 shows available options for Taguchi method. 


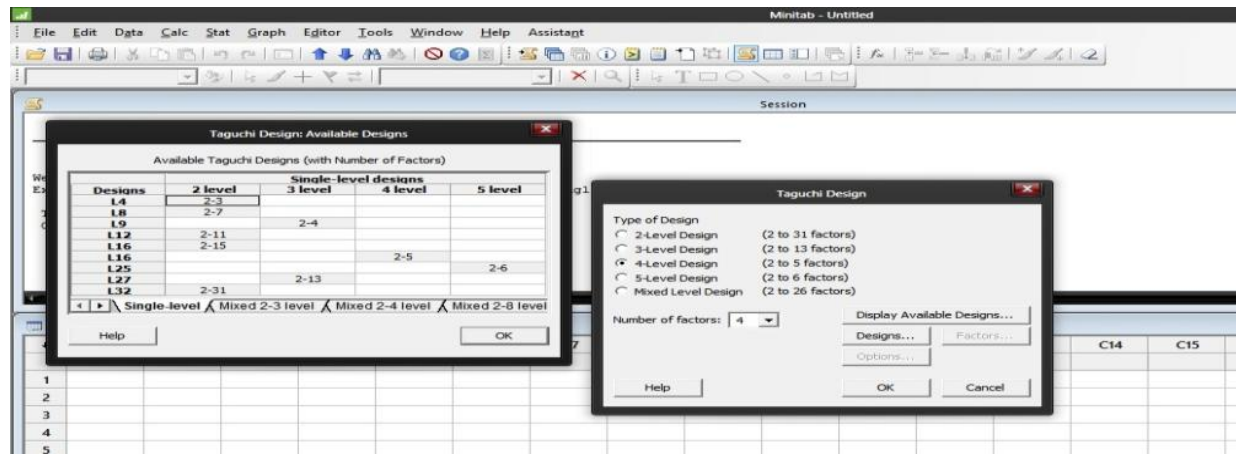

Fig 1.1 Available Options for Taguchi Method

Also, Fig 1.2 shows L16 Orthogonal Array with 4 Factors \& 4 Levels

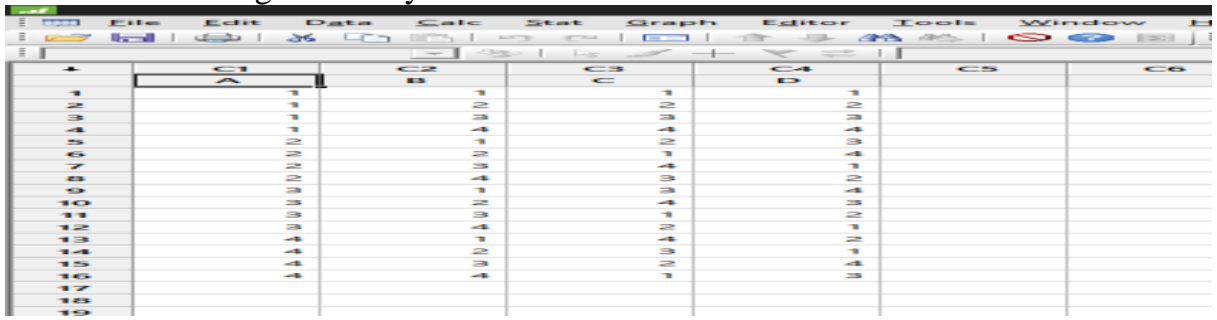

Fig 1.2- L16 Array

8. Assign Factors to $\mathrm{OA}$ and locate interactions:

In this step OA is created and iterations are decided.

L16 OA was created as per table 1.1 with Minitab 14 software

Table 1.1- L16 Orthogonal Array

\begin{tabular}{|l|l|l|l|}
\hline $\begin{array}{l}\text { Shot Size in } \\
\text { mm }\end{array}$ & $\begin{array}{l}\text { Shot Velocity in } \\
\mathbf{m} / \mathbf{s}\end{array}$ & $\begin{array}{l}\text { Impact Angle in } \\
\text { deg }\end{array}$ & $\begin{array}{l}\text { Shot Distance } \\
\text { mm }\end{array}$ \\
\hline 0.65 & 30 & 45 & 190 \\
\hline 0.65 & 40 & 65 & 120 \\
\hline 0.65 & 45 & 80 & 150 \\
\hline 0.65 & 60 & 85 & 190 \\
\hline 0.75 & 30 & 65 & 90 \\
\hline 0.75 & 40 & 45 & 150 \\
\hline 0.75 & 45 & 85 & 90 \\
\hline 0.75 & 60 & 80 & 120 \\
\hline 1 & 30 & 80 & 190 \\
\hline 1 & 40 & 85 & 150 \\
\hline 1 & 45 & 45 & 120 \\
\hline 1 & 60 & 65 & 90 \\
\hline 1.25 & 30 & 85 & 120 \\
\hline 1.25 & 40 & 80 & 90 \\
\hline 1.25 & 45 & 65 & 190 \\
\hline 1.25 & 60 & 45 & 150 \\
\hline
\end{tabular}

\section{Conduct the tests described by trials in OA:}

In this we have to take trials/iterations as per OA.

The trials are then done in FEM with help of LSDYNA Solver.

10. Analyze the Results of experimental trials:

The results are to be analyzed by Suitable Method.In this we have to maximize the residual stress and reduce the noise factors. So we have select larger is better form the $\mathrm{S} / \mathrm{N}$ ratio.

\section{Conduction Confirmation Experiment:}

The conduction of confirmation experiment is the last step in DOE. If conduction experiment gives desired results, the experimentation carried out is correct, otherwise reconstruct the experiment. In this if optimize result with larger the better $\mathrm{S} / \mathrm{N}$ ratio gives maximum residual stress compared with any other trial/iteration, the experiment carried out is said to be perfect. Also, fatigue life testing was carried out on specimen with optimized set of parameters.Columns of L16 Array levels 1234. 
Table 1.2 Parameter Control Factor Response Stress Factor

\begin{tabular}{|l|l|l|l|l|}
\hline Shot size & Shot Velocity & Impact Angle & Shot Distance & Stress \\
\hline 1 & 1 & 1 & 1 & 371.64 \\
\hline 1 & 2 & 2 & 2 & 49.70 \\
\hline 1 & 3 & 3 & 3 & 12.03 \\
\hline 1 & 4 & 4 & 4 & 8.22 \\
\hline 2 & 1 & 2 & 3 & 39.43 \\
\hline 2 & 2 & 1 & 4 & 1149.50 \\
\hline 2 & 3 & 4 & 1 & 12.56 \\
\hline 2 & 4 & 3 & 2 & 36.03 \\
\hline 3 & 1 & 3 & 4 & 31.80 \\
\hline 3 & 2 & 4 & 3 & 17.64 \\
\hline 3 & 3 & 2 & 2 & 585.64 \\
\hline 3 & 4 & 4 & 1 & 338.20 \\
\hline 4 & 1 & 3 & 2 & 26.96 \\
\hline 4 & 2 & 2 & 1 & 64.36 \\
\hline 4 & 3 & 4 & 339.86 \\
\hline
\end{tabular}

\subsubsection{Grey Relation Analysis for Multiple Responses (Compressive Residual Stresses, Surface Roughness)}

Grey relational analysis uses a specific concept of information. It defines situations with no information as black, and those with perfect information as white. However, neither of these idealized situations ever occurs in real world problems. In fact, situations between these extremes are described as being grey, hazy or fuzzy. Therefore, a grey system means that a system in which part of information is known and part of information is unknown. With this definition, information quantity and quality form a continuum from a total lack of information to complete information from black through grey to white. Since uncertainty always exists, one is always somewhere in the middle, somewhere between the extremes, somewhere in the grey area. Grey analysis then comes to a clear set of statements about system solutions. At one extreme, no solution can be defined for a system with no information. At the other extreme, a system with perfect information has a unique solution. In the middle, grey systems will give a variety of available solutions. Grey analysis does not attempt to find the best solution, but does provide techniques for determining a good solution, an appropriate solution for real world problems.

Table 1.3 Grey Relation Analysis Method for L16 Orthogonal Array

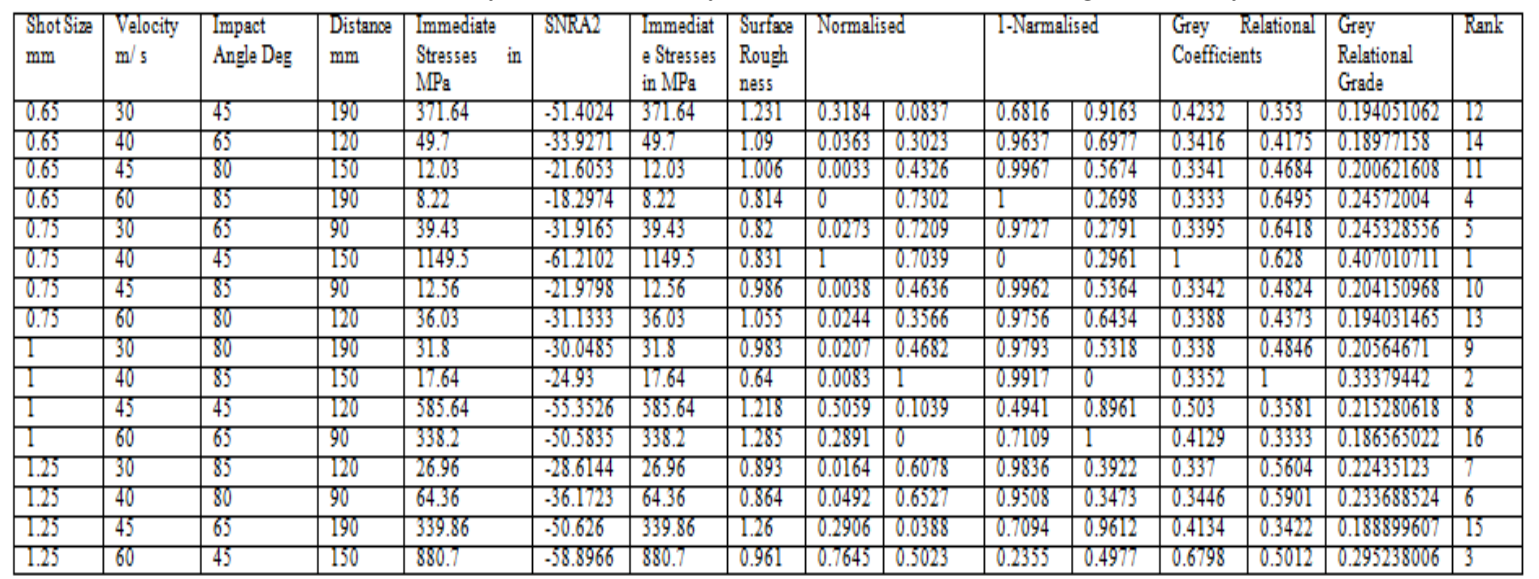

Table 1.4- Response Table for Signal to Noise Ratios

\begin{tabular}{|c|c|c|c|c|c|}
\hline $\begin{array}{c}\text { Shot Size } \\
\mathbf{m m}\end{array}$ & $\begin{array}{c}\text { Velocity } \\
\mathrm{m} / \mathbf{s}\end{array}$ & Impact Angle Deg & Distance mm & Grey Relational Grade & SNRA1 \\
\hline 0.65 & 30 & 45 & 190 & 0.19400 & -14.2417 \\
\hline 0.65 & 40 & 65 & 120 & 0.189772 & -14.4354 \\
\hline 0.65 & 45 & 80 & 150 & 0.200622 & -13.9524 \\
\hline 0.65 & 60 & 85 & 190 & 0.245720 & -12.1912 \\
\hline 0.75 & 30 & 65 & 90 & 0.245329 & -12.2050 \\
\hline 0.75 & 40 & 45 & 150 & 0.407011 & -7.8079 \\
\hline 0.75 & 45 & 85 & 90 & 0.204151 & -13.8010 \\
\hline 0.75 & 60 & 80 & 120 & 0.194031 & -14.2426 \\
\hline 1.00 & 30 & 80 & 190 & 0.205647 & -13.7376 \\
\hline 1.00 & 40 & 85 & 150 & 0.333794 & -9.5304 \\
\hline 1.00 & 45 & 45 & 120 & 0.215281 & -13.3399 \\
\hline 1.00 & 60 & 65 & 90 & 0.186565 & -14.5834 \\
\hline 1.25 & 30 & 85 & 120 & 0.224351 & -12.9814 \\
\hline 1.25 & 40 & 80 & 90 & -12.6273 & 0.233689 \\
\hline 1.25 & 45 & 65 & 190 & 0.188900 & -14.4754 \\
\hline 1.25 & 60 & 45 & 150 & 0.295238 & -10.5966 \\
\hline
\end{tabular}


Table 1.5 Taguchi Analysis: Grey Relation versus Shot Size mm, Velocity m/s, Impact Angle Deg, Distance $\mathrm{mm}$ Larger is better

\begin{tabular}{|l|l|l|l|l|}
\hline Level & $\begin{array}{l}\text { Shot Size } \\
\mathrm{mm}\end{array}$ & $\begin{array}{l}\text { Velocity } \\
\mathrm{m} / \mathrm{s}\end{array}$ & Impact Angle Deg. & $\begin{array}{l}\text { Distance } \\
\mathrm{mm}\end{array}$ \\
\hline 1 & -13.71 & -13.29 & -11.50 & -13.81 \\
\hline 2 & -12.01 & -11.10 & -13.92 & -13.75 \\
\hline 3 & -12.80 & -13.89 & -13.64 & -11.57 \\
\hline Delta & 1.69 & 2.79 & 2.43 & 2.24 \\
\hline Rank & 4 & 1 & 2 & 3 \\
\hline
\end{tabular}

Table 1.6 Response Table for Means

\begin{tabular}{|l|l|l|l|l|}
\hline Level & $\begin{array}{l}\text { Shot Size } \\
\mathrm{mm}\end{array}$ & Velocity m/s & $\begin{array}{l}\text { Impact Angle } \\
\text { Deg }\end{array}$ & $\begin{array}{l}\text { Distance } \\
\mathrm{mm}\end{array}$ \\
\hline 1 & 0.2075 & 0.2173 & 0.2779 & 0.2046 \\
\hline 2 & 0.2626 & 0.2911 & 0.2026 & 0.2059 \\
\hline 3 & 0.2353 & 0.2022 & 0.2085 & 0.2687 \\
\hline 4 & 0.2355 & 0.2304 & 0.2520 & 0.2618 \\
\hline Delta & 0.0551 & 0.0888 & 0.0753 & 0.0641 \\
\hline Rank & 4 & 1 & 2 & 3 \\
\hline
\end{tabular}

Table 1.7 Analysis of Variance

\begin{tabular}{|l|l|l|l|l|l|}
\hline Source & DF & Adj SS & AdjMS & F-Value & P-Value \\
\hline Shot Size mm & 3 & 0.006070 & 0.002023 & 2.68 & 0.220 \\
\hline Velocity m/ s & 3 & 0.018198 & 0.006066 & 8.03 & 0.060 \\
\hline Impact Angle Deg & 3 & 0.015513 & 0.005171 & 6.85 & 0.074 \\
\hline Distance mm & 3 & 0.014521 & 0.004840 & 6.41 & 0.081 \\
\hline Error & 3 & 0.056568 & 0.000755 & & \\
\hline Total & 15 & 0.056568 & & & \\
\hline
\end{tabular}

Table 1.8 Model Summaries

\begin{tabular}{|l|l|l|l|}
\hline S & R-sq & R-sq(adj) & R-sq(pred) \\
\hline 0.0274802 & $96.00 \%$ & $79.98 \%$ & $90.00 \%$ \\
\hline
\end{tabular}

Regression Equation for Coefficient

Grey Relational Grade $=0.23526-0.0277$ Shot Size mm_0. 65+0.0274 Shot Size mm_0.75+0.0001 Shot Size mm_1.00+ 0.0003 Shot Size mm_1.25-0.0179 Velocity m/ s_30+ 0.0558 Velocity m/ s_40-0.0330 Velocity m/ s_45-0.0049 Velocity m/ s_60+ 0.0426 Impact Angle deg_45 0.0326 Impact Angle deg_65-0.0268 Impact Angle deg_80+ 0.0167 Impact Angle deg_85- 0.0306 Distance mm_90- 0.0294 Distance mm_ 120+ 0 .0335 Distance $\mathrm{mm} \_150+0.0266$ Distance $\mathrm{mm} \_190$.

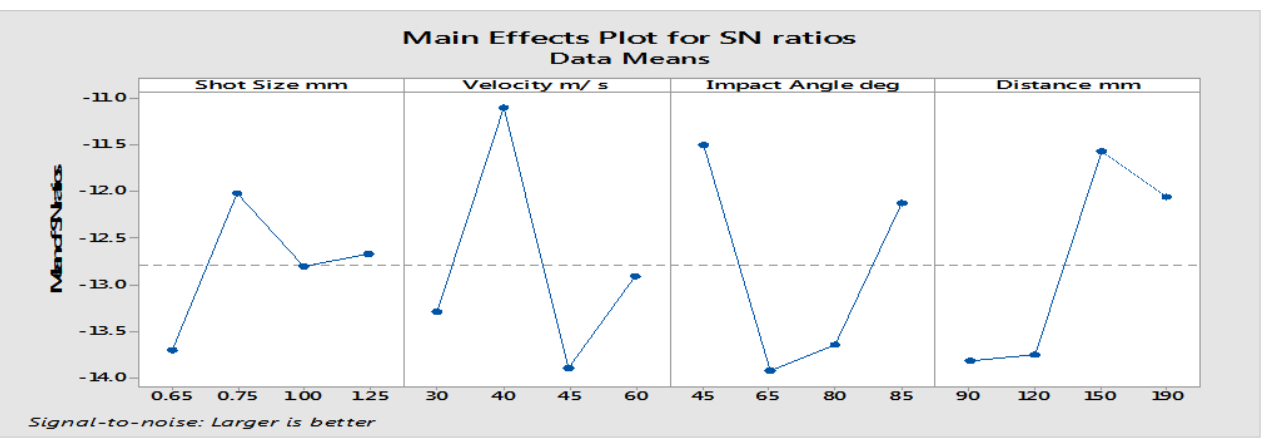

Fig 1.3- Main Effects Plot for S/N Ratios Data Means

Table 1.9- Table of Optimized Value

\begin{tabular}{|l|l|l|l|l|}
\hline Level & Shot Size $\mathrm{mm}$ & Velocity $\mathrm{m} / \mathrm{s}$ & Impact Angle Deg & Distance $\mathrm{mm}$ \\
\hline Optimized Value & 0.75 & 40 & 45 & 150 \\
\hline
\end{tabular}

Regression Equation for grade

Grey Relational Grade $=0.326-0.91$ Shot Size $\mathrm{mm}-0.0052$ Velocity $\mathrm{m} / \mathrm{s}$

+0.00128 Impact Angle deg + 0.00447 Distance mm

+0.0111 Shot Size $\mathrm{mm} *$ Velocity $\mathrm{m} / \mathrm{s}$

+0.01022 Shot Size mm*Impact Angle deg

- 0.00248 Shot Size mm*Distance mm 
- 0.000115 Velocity m/s*Impact Angle deg

+0.000036 Velocity $\mathrm{m} / \mathrm{s} *$ Distance $\mathrm{mm}$

- 0.000040 Impact Angle deg*Distance mm

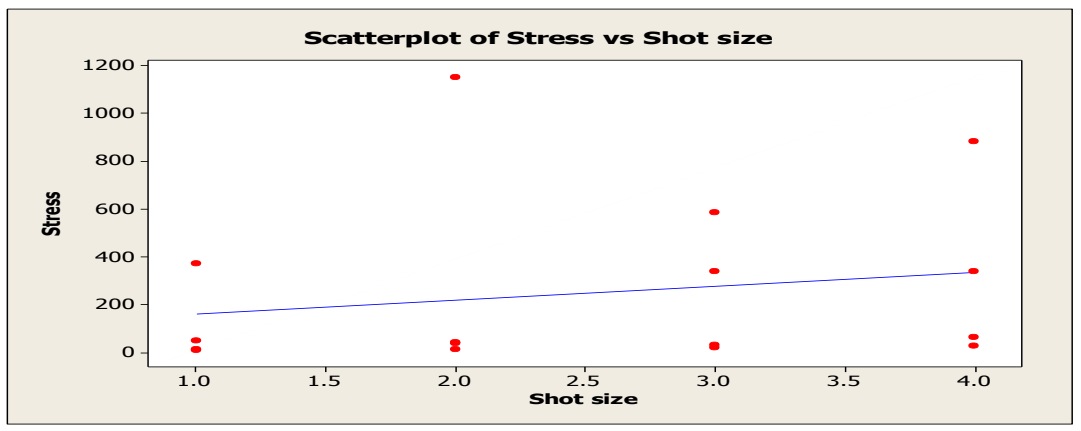

Graph 1.1:- Scatter Plot of Stress Vs Shot Size

Graph 1.1 it is Graph Between stress vs shot size gives the scattering relationship from both sides of mean line.

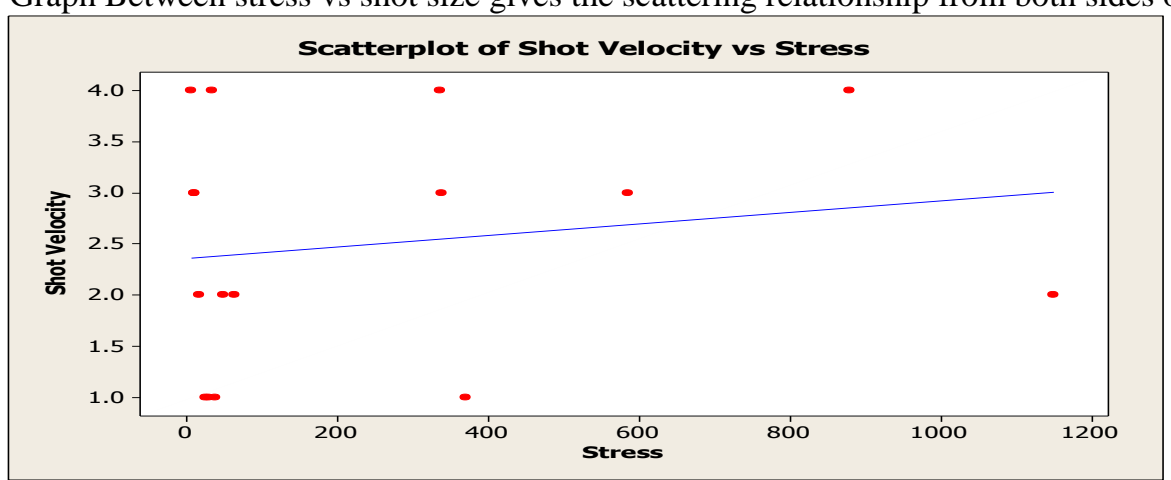

Graph 1.2:- Scatter Plot of Shot velocities Vs Stress

Graph 1.2 Shows Graph between Shot velocities Vs Stress gives the scattering relationship from both sides of mean line.

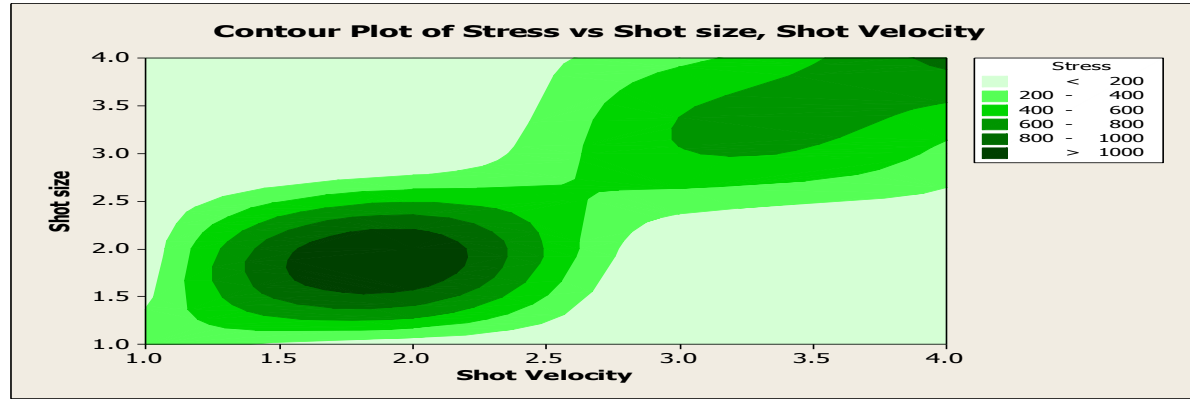

Graph 1.3:- Counter Plot of Stress Vs Shot Size Vs Shot Velocity

Graph 1.3 shows Graph between Stresses vs. shot size vs. shot velocity gives the scattering nonlinear relationship.

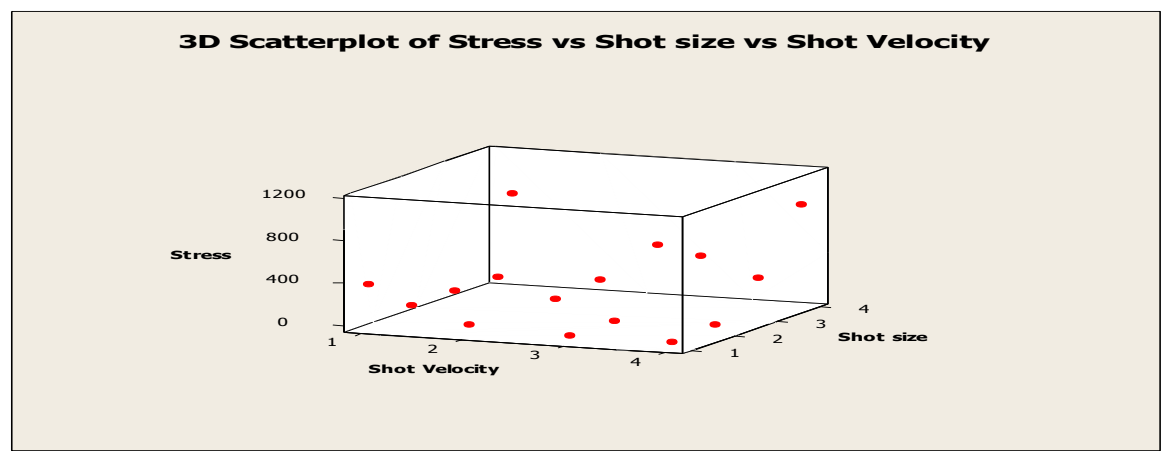

Graph 1.4- 3 D Scatter Plot of Stress Vs Shot Size Vs Shot Velocity 
Graph 1.4 shows graph between $3 \mathrm{D}$ scatter plot of stress vs shot size vs. shot velocity gives the scattering nonlinear relationship.

\subsection{Simulated Annealing}

It is the Metropolis algorithm which can be used to generate a sequence of solutions of a combinatorial optimization problem. Simulated annealing solves optimization problems using a probabilistic search algorithm that mimics the physical process of annealing, in which a material is heated and then the temperature is slowly lowered to decrease defects, thus minimizing the system energy. By analogy each iteration of a simulated annealing algorithm seeks to improve the current minimum by slowly reducing the extent of the search. The simulated annealing algorithm accepts all new points that lower the objective, but also, with a certain probability, points that raise the objective. By accepting points that raise the objective, the algorithm avoids being trapped in local minima in early iterations and is able to explore globally for better solutions.

\subsubsection{Simulated Annealing Solver}

Simulated annealing allows you to solve unconstrained or bound-constrained optimization problems and does not require that the functions be differentiable or continuous. From the command line or Optimization application you can use toolbox functions to:

1. Solve problems using adaptive simulated annealing, Boltzmann annealing, or fast annealing algorithms

2. Create custom functions to define the annealing process, acceptance criteria, temperature schedule, plotting functions, simulation output, or custom data types

3. Perform hybrid optimization by specifying another optimization method to run at defined intervals or at normal solver termination

Objective Function = @GRG;

\section{Results}

Objective Function Value: 0.42378173411230374
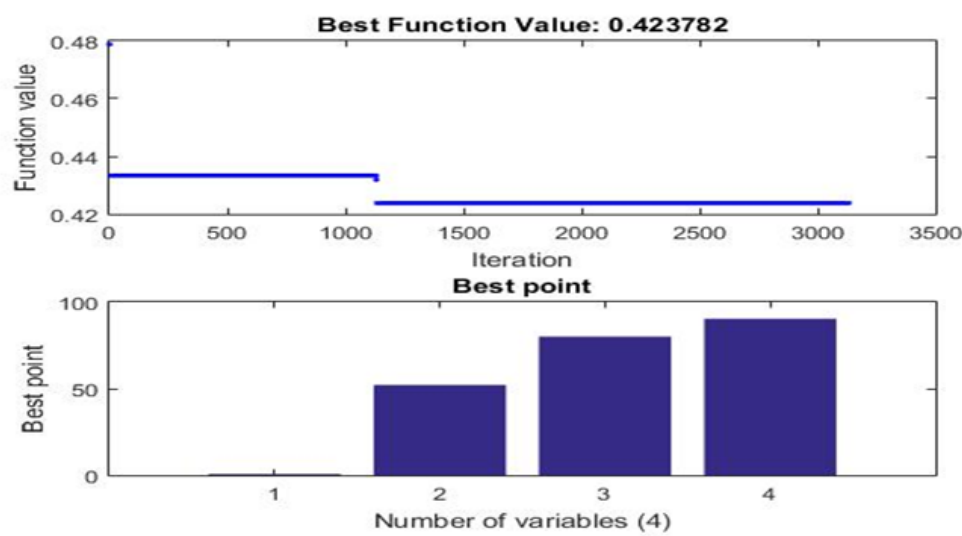

Graph 1.5: - Graph Between Fitness Value Vs Iteration Generation Best Point Vs Number of Variables, Current Point Vs Number of Variables

\subsection{Genetic Algorithm}

\subsubsection{Introduction}

Genetic Algorithms (GA) are direct, parallel, stochastic method for global search and optimization, which imitates the evolution of the living beings, described by Charles Darwin. GA is part of the group of Evolutionary Algorithms (EA). The evolutionary algorithms use the three main principles of the natural evolution: reproduction, natural selection and diversity of the species, maintained by the differences of each generation with the previous. The selection principle is applied by using a criterion, giving an evaluation for the individual with respect to the desired solution. The best-suited individuals create the next generation. The large variety of problems in the engineering sphere, as well as in other fields, requires the usage of algorithms from different type, with different characteristics and settings Genetic Algorithms (GA) are direct, parallel, stochastic method for global search and optimization, which imitates the evolution of the living beings, described by Charles Darwin. GA is part of the group of Evolutionary Algorithms (EA).

1. Generate initial population - in most of the algorithms the first generation is randomly generated, by selecting the genes of the chromosomes among the allowed alphabet for the gene. Because of the easier computational procedure it is accepted that all populations have the same number $(N)$ of individuals.

2. Calculation of the values of the function that we want to minimize of maximizes.

3. Check for termination of the algorithm - as in the most optimization algorithms, it is possible to stop the genetic optimization by: 
4. Value of the function - the value of the function of the best individual is within defined range around a set value. It is not recommended to use this criterion alone, because of the stochastic element in the search the procedure, the optimization might not finish within sensible time.

5. Maximal number of iterations - this is the most widely used stopping criteria. It guarantees that the algorithms will give some results within some time, whenever it has reached the Extremes or not.

6. Stall generation - if within initially set number of iterations (generations) there is no improvement of the value of the fitness function of the best individual the algorithms stops.

7. Selection - between all individuals in the current population are chose those, who will continue and by means of crossover and mutation will produce offspring population. At this stage elitism could be used the best $n$ individuals are directly transferred to the next generation. The elitism guarantees, that the value of the optimization function cannot get worst (once the Extremum is reached it would be kept).

8. Crossover - the individuals chosen by selection recombine with each other and new

9. Individuals will be created. The aim is to get offspring individuals, which inherit the best possible combination of the characteristics (genes) of their parents.

10. Mutation - by means of random change of some of the genes, it is guaranteed that even if none of the individuals contain the necessary gene value for the Extremism, it is still possible to reach the Extremes.

11. New generation - the elite individuals chosen from the selection are combined with

12. Those who passed the crossover and mutation, and form the next generation.

1.4.2 Results

Objective Function = @GRG;

Objective Function Value: 0.4788149999999999
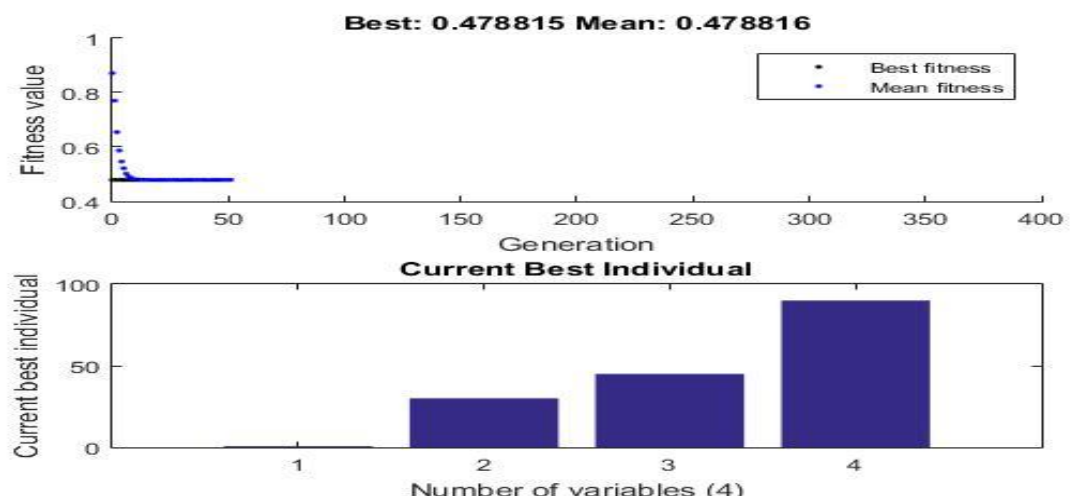

Graph: 1.6-Graph between Fitness Value Vs Number of Variables Generation Current Best Individuals 1.5 Discussion of Results

By comparing results of optimization methods represent in tab 4.12 which is acceptable

Table 4.12- Comparing Results Grades of Optimization Methods

\begin{tabular}{|l|l|l|l|l|l|}
\hline Sr. & Optimization Method & $\begin{array}{l}\text { Grey } \\
\text { Relation } \\
\text { No. }\end{array}$ & $\begin{array}{l}\text { Genetic } \\
\text { Algorithm } \\
\text { Saguchi } \\
\text { Shot Size mm Shot velocity } \\
\text { m/s Impact Angle Deg. } \\
\text { Distance mm }\end{array}$ & $\begin{array}{l}\text { Simulated } \\
\text { Annealing } \\
\text { Method } \\
\text { Method }\end{array}$ & \\
\hline 01 & Grades & 0.40 & 0.4237 & 0.478815 & $\%$ \\
\hline
\end{tabular}

\section{Conclusions}

1. Shot size varied from $0.65 \mathrm{~mm}$ to $1.25 \mathrm{~mm}$. 4 Levels were selected as $0.65,0.75,1.00,1.25 \mathrm{~mm}$. We can get from Signal to Noise ratio that the residual stress increases as the shot size increases for PMG Cover of $\mathrm{Al} 2024$ alloy.

2. Shot Velocity varied from $30 \mathrm{~m} / \mathrm{s}$ to $60 \mathrm{~m} / \mathrm{s}$ in 4 levels as $30,40,45$ and $60 \mathrm{~m} / \mathrm{s}$. From Signal to Noise ratio that the residual stress increases as the shot velocity increases for PMG Cover of Al 2024 alloy.

3. Shot Impact Angles were selected as $45^{\circ}, 65^{\circ}, 80^{\circ} \& 85^{\circ}$ as 4 levels. The $\mathrm{S} / \mathrm{N}$ ratio graphs clearly indicates that as angle moves towards $90^{\circ}$ the residual stress induced is going to decrease for PMG Cover of Al 2024.

4. Shot distances were chosen as $90,120,150,190 \mathrm{~mm}$ as 4 levels of experimentation. For first it has maximum value of residual stress for $150 \mathrm{~mm}$. for PMG Cover of Al 2024 alloy.

5. The optimum solution for maximizing the residual stress is Shot Size $0.75 \mathrm{~mm}$, Shot Velocity $40 \mathrm{~m} / \mathrm{s}$, Impact angle $45^{\circ}$ and Shot Distance $150 \mathrm{~mm}$. Residual stress at this iteration is $338.00 \mathrm{MPa}$. 
6. For the particular given set of values of parameters Shot Size $0.75 \mathrm{~mm}$, Shot Velocity $40 \mathrm{~m} / \mathrm{s}$, Impact angle $45^{\circ}$ and Shot Distance $150 \mathrm{~mm}$ is the optimum parameter set. The deflection for this was 135.0 microns. Immediate stress was $1100 \mathrm{MPa}$. Residual stress is 338.0 MPa for this.

7. The behavior of the parameters can be seen by $\mathrm{S} / \mathrm{N}$ ratio graphs with strategy Larger the better.

8. Grades are getting by Grey Relation Method Genetic Algorithm Simulated Annealing 0.40, 0.43, and 0.47 respectively.

9. Acceptable Percentage of Grade variations in optimization methods comparisons is up to $14.02 \%$.

\section{References}

[1] George Leghorn, "The story of shot peening”, A.S.N.E. Journal November 1957, pp 653- 666.

[2] Y.F. Al-Obaid," Shot Peening Mechanics: experimental and theoretical analysis", Mechanics of Materials, 1995

[3] S.Wang, Y.Li,m.Yao, R.Wang," Compressive residual stress introduced by shot peening”, Journal of Materials Processing Technology, 1998

[4] Romero,Rios,Fam,Levers," Optimization of the shot peening process in terms of fatigue resistance", Conf Proc: ICSP-7 ,Warsaw,Poland, 1999

[5] Guagliano M, Vergani L, Bandini M, Gili F. "An approach to relate the shot peening parameters to the induced residual stresses", Conf Proc: ICSP-7, Warsaw, Poland, 1999.

[6] S. Curtis, E.R. de los Rios, C.A. Rodopoulos, A. Levers," Analysis of the effects of controlled shot peening on fatigue damage of high strength aluminum alloys", International Journal of Fatigue, 2003

[7] Renaud, “Optimization of Shot peening Parameters”, Conf Proc: ICSP-8 ,Garmisch-Partenkirchen, Germany, 2002

[8] R. Menig, V. Schulze, D. Lohe, O. Vohringer, "Shot peening plus subsequent short time annealing-a way to increase residual stress stability and alternating bending strength of AISI 4140", Society of automotive engineers, 2002

[9] Rodopoulos, Curtis, Rios, SolisRomero," Optimization of the fatigue resistance of 2024-T351 aluminum alloys by controlled shot peening- methodology, results and analysis", International Journal of Fatigue, 2004

[10] Ludian and Wanger," Coverage Effects in Shot Peening of Al 2024-T4", Conf Proc: ICSP-9 , Paris, France ,2005

[11] A.Inoue,T.Sekigawa,K.Oguri," Fatigue property enhancement by fine particle shot peening for aircraft aluminum parts", Conf Proc: ICSP-10, Tokyo, Japan ,2008. 